\title{
A Research on Business Information System and Informatization
}

\author{
Fangyuan Liu* \\ Birmingham Business School, University of Birmingham, Birmingham B15 2TT, UK \\ *Corresponding author: Fangyuan Liu, 1005239297@qq.com
}

\begin{abstract}
Based on the analysis of commercial economic informatization, this paper puts forward the abstract model of commercial information system, which comprises of the environmental system, business system, monitoring system, and emergency system. In addition, it also involves the strain cycle, monitoring cycle, and business cycle. In order to promote a better development in the business economic model under the background of informatization, commercial enterprises should improve their understanding, develop and continuously optimize business economic informatization, as well as create an efficient and safe network security environment for the future.
\end{abstract}

Keywords: Business economic informatization; Business information system; Informatization; Business economic model

Publication date: August 2021; Online publication: August 30, 2021

\section{Introduction}

Along with the continuous development and progress of commercial economy, the importance of information construction is becoming much more prominent. With the support of information construction, the development of the commercial economy in China can be further promoted. With the innovation of the commercial economic development model, the important role of information cannot be disregarded. Nowadays, informatization has developed into an important and a main channel of China's business economic model. Along with the improvement of the modernization level of the main channel, commodity circulation also shows an accelerating trend. Increasing the informatization construction effort allows the maintenance of a coordinated development of commercial economy. In the process of accelerating the speed of informatization, the production and development of commerce as well as the speed of capital turnover are also accelerating. On the whole, China's national economy is developing at a very fast pace. Therefore, in the current situation, it is necessary to build a modern business information system to realize information development and drive the progress of business economy from the essential level.

\section{Commercial economic informatization}

Commercial economic informatization refers to the use of information technology and means with modern development characteristics, such as the network, computer, and communication facilities, in addition to the effective integration of various interrelated enterprise information resources under the commercial economic model in order to achieve a profound development of commercial economic information resources, form a strong impetus to the effectiveness, rationality, and feedback of commercial economy, as well as strengthen the core competitiveness among enterprises based on the commercial economic model from the essential level to create greater economic benefits for enterprises. Under the macro background of information development, the development of the business economic model can realize the transformation from a traditional model to a modern trade direction, which is conducive to improve the modernization 
level of business economy and trade effectively. In addition, with that, it is also possible to optimize trade means and methods.

\section{Business information system model}

Commercial enterprises should formulate and modify commodity sales countermeasures based on consumers' purchase trends and the market conditions. In addition, they need to understand the situation of their competitors. All these require enterprises to accumulate market information, especially those pertaining to other commercial enterprises, and engender effective responses based on those information in order to achieve a good response in fierce market competition. In this process, enterprises can abstract the relationship between departments and institutions into logistics flow, capital flow, and information flow. The aforementioned logistics flow refers to commodity flow, which flows from suppliers to commercial enterprises and subsequently to consumers. The capital flow direction is opposite to the logistics flow direction, and they are accompanied by information flow. Therefore, a business information system should effectively manage enterprise information, provide guarantee for the smooth flow of internal logistics and capital, then realize rapid and accurate responses to the external environment. Figure 1 shows the abstract model of a business information system.

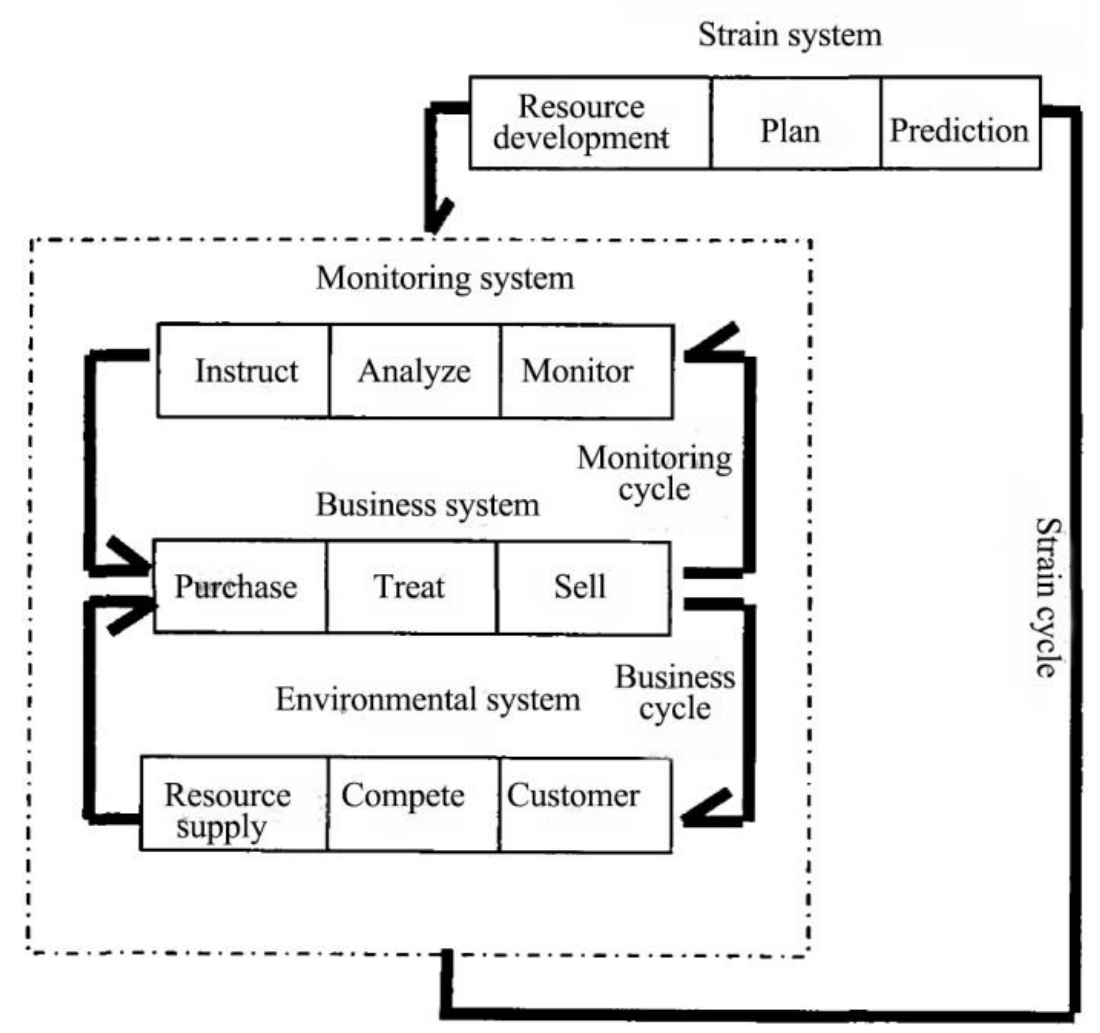

Figure 1. Abstract model of a business information system

The abstract model involves four subsystems: environment system, business system, monitoring system, and emergency. Among them, the environment system is used to deal with the internal and external relations of the enterprise; the main function of the business system is to process the main business of the enterprise, which involves purchase, sales, and inventory transfer; the function of the monitoring system is to monitor the actual operation of commodities, issue instructions based on corresponding changes, and adjust the business system; the strain system is to monitor the situation of the whole enterprise and for market analysis. 
There are three cycles involved in the above four systems: strain cycle, monitoring cycle, and business cycle. Among them, the strain cycle takes into consideration of the overall situation of the enterprise as the starting point, analyzes the medium-term and long-term objectives, formulates specific plans, as well as provides corresponding guidance for the operation and development of the enterprise; the monitoring cycle involves the business and monitoring systems, in which it focuses on short-term behavior and emphasizes the enterprise's adaptation to the market; together, the business system and environment system form a business cycle, which is principally used to realize the specific objectives of the enterprise.

\section{Development strategy of the commercial economic model based on information development}

\subsection{Raising awareness, developing, and continuously optimizing commercial economic informatization}

Under the macro background of computer and information technology, China's productive forces are also developing in the direction of informatization. In order to realize the construction of an effective commercial information system, commercial enterprises need to use information as the trend to seek continuous development and optimization so as to adapt well to the requirements of the new era, strengthen market competitiveness, and at the same time, open up a broader network market as well as expand the scale of online transactions. In the development process of commercial economic informatization, it is necessary to perfect the construction of e-commerce platforms, share information resources on these platforms, and innovate the commercial economic model. Enterprises should take into consideration of their actual situations for a breakthrough in the development of information, purposefully change their own management mode, as well as improve the management level and quality.

\subsection{Creating an efficient and secure network security environment}

In regard to the general trend of information development, the issue of enterprise information security has become the new research focus and direction in the development of China's commercial economy. In the construction of the network system, there should be a comprehensive consideration of the security of information. The whole network system should be used as the starting point. Several security strategies which are used by the network system should be combined effectively and the effectiveness of network security prevention based on the general trend of information development should be continuously improved. In addition, the risk incidence should be minimized while avoiding unsafe factors, and the continuous innovation of the business economic model under the background of information development should be strongly promoted. Eventually, the overall benefits of business economy would be brought into play.

\section{Disclosure statement}

The author declares that there is no conflict of interest.

\section{References}

[1] Wang K, 2021, Research on the Feasibility of Establishing a Business Information System in Line with Network Security Standards. Network Security Technology \& Application, (3): 97-8.

[2] Su QQ, Jia YL, Wu YR, 2017, Analysis of Informatization Business Model and Enterprise Management Innovation. Fast Reading, (3): 227. 